\title{
MAPEAMENTO DE SUPERFÍCIES IMPERMEÁVEIS NA REGIÃO METROPOLITANA DE SÃO PAULO UTILIZANDO FOTOGRAFIAS AÉREAS E IMAGENS LANDSAT-8 OLI
}

\author{
Fernando Shinji Kawakubo $^{(a)}$, Marcos Henrique Martins ${ }^{(b)}$, Rubia Gomes Morato ${ }^{(c)}$ \\ (a) Departamento de Geografia /Faculdade de Filosofia, Letras e Ciências Humanas, Universidade de São Paulo, \\ Email fsk@usp.br \\ (b) Departamento de Geografia /Faculdade de Filosofia, Letras e Ciências Humanas, Universidade de São Paulo, \\ Email marcos.henrique.martins@usp.br. \\ (c) Departamento de Geografia /Faculdade de Filosofia, Letras e Ciências Humanas, Universidade de São Paulo, \\ Email rubiagm@usp.br
}

\section{Eixo: GEOTECNOLOGIAS E MODELAGEM ESPACIAL EM GEOGRAFIA FÍSICA}

\begin{abstract}
Resumo
Um dos efeitos mais marcantes da urbanização é o aumento de superfícies impermeáveis. A impermeabilização do solo traz vários impactos negativos ao ambiente, como mudanças na infiltração e escoamento superficial das águas, aumento da temperatura do ambiente, deterioração da qualidade da água etc. Em razão de sua importância, o presente estudo tem como objetivo mapear as superfícies impermeáveis na Região Metropolitana de São Paulo (RMSP) utilizando informações combinadas de ortofotos digitais e imagens multiespectrais do sensor Operational Land Imager (OLI) a bordo do satélite Landsat-8. Para a execução do trabalho, os seguintes procedimentos foram adotados: (1) seleção e definição de áreas de controle utilizando fotografias aéreas; (2) geração do índice Normalized Intensity Vegetation Index (NDVI) utilizando as bandas do vermelho e infravermelho próximo; (3) geração do modelo de regressão para a estimativa espacial de superfícies impermeáveis. Os resultados alcançados foram avaliados qualitativamente e por meio de testes estatísticos.
\end{abstract}

Palavras chave: Escoamento superficial, NDVI, regressão linear, validação

\section{Introdução}

As superfícies impermeáveis correspondem às áreas onde não ocorre infiltração da água no solo em razão da impermeabilização provocada pela pavimentação do sistema viário, calçadas, estacionamentos, construção de habitações, fábricas, galpões etc. Apesar de constituir uma das expressões mais importantes da urbanização (ARNOLD; GIBBONS, 1996) (CANTERS et al., 2006), as superfícies impermeáveis não possuem apenas origem antropogênica; afloramentos rochosos e solos muito compactados também constituem "superfícies naturais" impermeáveis, ou ao menos, semipermeáveis.

Além de se constituir como um importante indicador do grau de urbanização de uma determinada unidade geográfica, as superfícies impermeáveis também exercem um papel crucial na qualidade ambiental do entorno (ARNOLD; GIBBONS, 1996). A princípio, a simples disposição de materiais em superfície que impedem ou dificultam a infiltração da água no solo não trazem necessariamente impactos negativos à 
sociedade e ao meio ambiente. Todavia, uma série de processos danosos é desencadeada pelo aumento indiscriminado de superfícies impermeáveis.

Talvez o impacto mais direto do aumento da impermeabilização do solo, especialmente nos grandes centos urbanos, seja as mudanças no regime hidrológico com a consequente redução da infiltração da água no solo e do aumento no escoamento superficial. De acordo com Tocci (1997), a redução da infiltração impacta a recarga do lençol freático e diminui a vazão dos rios, especialmente nas épocas de seca. Já o aumento do escoamento superficial potencializa os problemas de magnitude e frequência de inundação. Isto porque o volume de água que deixa de infiltrar no solo permanece na superfície e é escoado por condutos pluviais que amentam a velocidade do fluxo de deslocamento e as vazões máximas, antecipando assim os picos no tempo (TOCCI, 1997).

Outro problema desencadeado pelo aumento de superfícies impermeáveis é a alteração no fluxo de calor latente e sensível, causando ilhas de calor (CHANGNON, 1992). Materiais como asfalto, concreto e amianto, por exemplo, possuem propriedades de absorver grande quantidade de energia solar, que então é liberada na forma de calor. Como consequência, há uma forte relação linear positiva entre aumento de superfície impermeável e aumento da temperatura do ambiente e uma relação inversa com a densidade de vegetação (YUAN; BAUER, 2007).

A deterioração da qualidade da água é um outro exemplo que aflige a sociedade e que possui relação com o tema aqui tratado. Infelizmente, também se observa uma relação bastante estreita envolvendo a qualidade da água e a porcentagem de cobertura impermeável presente nas bacias hidrográficas (ARNOLD; GIBBONS, 1996). Não é nenhuma surpresa admitir que a manutenção da qualidade e/ou integridade de uma bacia hidrográfica depende de um nível mínimo de proteção que resguarde as suas características ditas naturais. Neste sentido, estudos realizados por (SCHUELER, 1994) revelam que em muitas áreas geográficas, a degradação do ambiente ocorre em níveis relativamente baixos de impermeabilização do solo, que podem variar de 10 a 20\%. A impermeabilização nestes casos age no sentido de aumentar o transporte de nutrientes (nitrogênio e fósforo), de contaminantes tóxicos e de agentes patogênicos para os rios, córregos e lagos.

Uma característica importante das superfícies impermeáveis é que elas não se manifestam de maneira pontual no espaço, e em razão disto, o planejamento constitui o mecanismo mais eficiente de contenção e/ou mitigação dos danos decorrentes de um uso da terra inadequado ou de pouca conformidade com o seu entorno.

Felizmente é possível mapear as superfícies impermeáveis utilizando técnicas de sensoriamento remoto (WENG, 2007). A forma mais tradicional de mapear as superfícies impermeáveis é utilizando fotografias 
aéreas de voo baixo. O detalhe geométrico proporcionado pelas fotografias aéreas associada com a utilização das técnicas de fotointerpretação (PAINE; KISER, 2003) permitem que o analista, em um primeiro momento, identifique os diferentes tipos de uso da terra e cobertura vegetal, e em uma etapa posterior, relacione os diferentes tipos de usos com a disposição de materiais impermeáveis. Dentre os principais empecilhos da utilização das fotografias aéreas, é possível destacar: a limitação do ponto de vista de recobrimento temporal; o alto custo quando comparadas com as imagens de satélite; a subjetividade e demora na elaboração dos mapeamentos.

As imagens de satélite, por sua vez, possuem o atrativo de cobrir grandes áreas, fornecendo então uma visão integral da paisagem analisada, com alta frequência temporal, coletando informações em diferentes partes do espectro eletromagnético e fazendo uso muitas vezes de técnicas de extração de informação automatizada.

Diante da importância da obtenção de informações acerca de superfícies impermeáveis, o presente estudo tem como objetivo mapear as áreas impermeáveis na Região Metropolitana de São Paulo (RMSP) utilizando uma metodologia que combina informações geradas por diferentes produtos de sensoriamento remoto. Fotografias aéreas de alta resolução espacial e imagens do sensor Operational Land Imager (OLI) do satélite Landsat-8 foram utilizadas para a estimativa contínua de impermabilização do solo. A realização deste estudo justitica-se pela importância de aprimorar metodologias que visem o calculo de superfície impermeáveis utilizando produtos de média resolução espacial (de 10 a 100m).

\section{Metodologia}

Foram utilizados os seguintes materiais para a realização da pesquisa:

- Ortofotos digitais disponibilizadas pela Empresa de Planejamento Metropolitano (EMPLASA) com resolução Ground Sample Distance (GSD) de 45centímetros (reamostrada para 1 metro no processo de ortorretificação), referente ao voo dos anos de 2010/2011.

- Imagens ortorretificadas do satélite Landsat-8, sensor OLI disponíveis no sítio da U.S. Geological Survey (USGS) do ano de 2015.

- Software ArcGIS (ESRI) disponível no Laboratório de Aerofotogeografia e Sensoriamento Remoto (Lasere) do Departameto de Geografia da Faculdade de Filosofia, Letras e Ciências Humanas (DG-FFLCH) da Universidade de São Paulo (USP). 
Conforme pode ser observado na Figura 1, a presente pesquisa procura explorar o sinergismo de informações obtidas a partir da alta resolução espacial das fotografias aéreas e o amplo recobrimento do terreno das imagens orbitais multiespectrais.

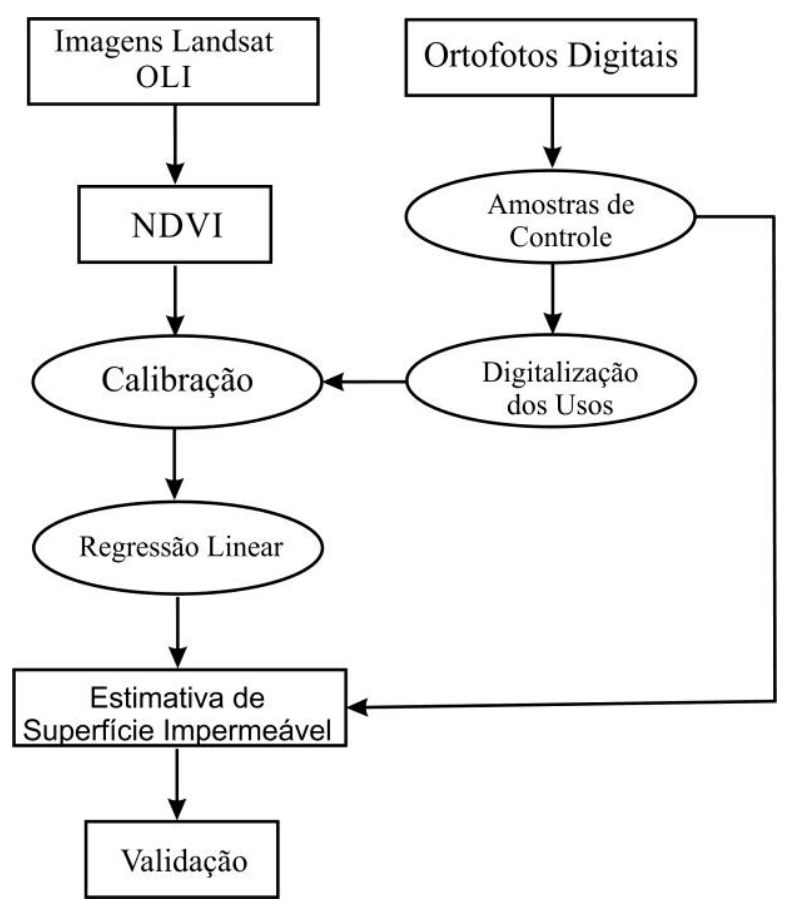

Figura 1 - Fluxograma dos principais procedimentos adotados na pesquisa.

A primeira etapa consistiu na seleção e definição das amostras de controle. Utilizando as informações detalhadas das ortofotos digitais, classificações de uso da terra e cobertura vegetal foram realizadas visualmente com base nos elementos de reconhecimento da fotointerpretação (CERON; DINIZ, 1966, PAINE; KISER, 2003). É importante esclarecer que a interpretação do uso da terra e cobertura vegetal não foi feita para toda a área de estudo, mas em apenas algumas áreas previamente selecionadas. Tomou-se o cuidado de selecionar apenas áreas que não sofreram alterações entre os anos de 2010 e 2015. Foram digitalizadas 5000 amostras de controle, sendo que para cada localidade, a porcentagem de superfície impermeável foi calculada tomando como referência a resolução espacial das imagens OLI (30 metros no terreno ou 900 metros quadrados).

Para realizar o calculo de estimativa de superfície impermeável foram utilizados 4500 amostras de controle, que foram confrontados com os valores dos pixels das imagens OLI. As imagens foram previamente convertidas para valores de reflectância no topo da atmosfera com o objetivo de melhor caracterizar o comportamento espectral dos objetos. Em razão das áreas impermeáveis apresentarem 
comportamento inverso à abundância da vegetação, o índice de vegetação Normalized Density Vegetation Index (NDVI) foi utilizado como variavel explicativa do modelo de regressão linear. O NDVI foi calculado utilizando a seguinte formula:

$\mathrm{NDVI}=\mathrm{B} 5-\mathrm{B} 4 / \mathrm{B} 5+\mathrm{B} 4$

Onde:

B5 é a banda do canal infravermelho próximo

B4 é a banda do canal vermelho

Os valores de NDVI variam de -1 a 1 . A interpretação desse índice é feita da seguinte forma: valores negativos e próximos de 0 representam áreas com ausência de vegetação, enquanto valores positivos correspondem às áreas vegetadas cuja intensidade aumenta com o incremento do índice. Assim, com o objetivo de evitar valores negativos na fase de modalgem, os referidos valores foram transformados em zero.

Teoricamente, a "zona" e acerto da modelagem restringe os resultados dentro do intervalo de valores de $0 \%$ (superficie totalmente permeável) a $100 \%$ (superficie totalmente impermeável). Os valores que extrapolam este intervalo podem ser considerados fiscamente inconsistentes e foram tratados de uma forma bastante simples reposicionando os valores extrapolados. Ou seja, valores negativos foram transformados em 0 e valores acima de 1 , em 1 .

A fim de avaliar os erros de estimativa, procedeu-se a validação do modelo gerado utilizando as 500 amostras de controle separadas previamente. Duas análises foram realizadas: ajuste por meio da reta de regressão com o objetivo de verificar a correlação existente entre os dados; a subtração do valor estimado com o real, sendo que para um ajuste perfeito, a população segue uma distribuição normal com média zero.

\section{Resultados}


Os resultados da modelagem relevaram uma boa correlação entre os valores de NDVI e as referências de impermeabilização do solo calculadas a partir das fotografias aéreas. A reta de regressão construida com ajuste linear obteve $\mathbf{R}^{2}=0,75 \operatorname{com} p<0,05$, conforme ilustra a Figura 2.

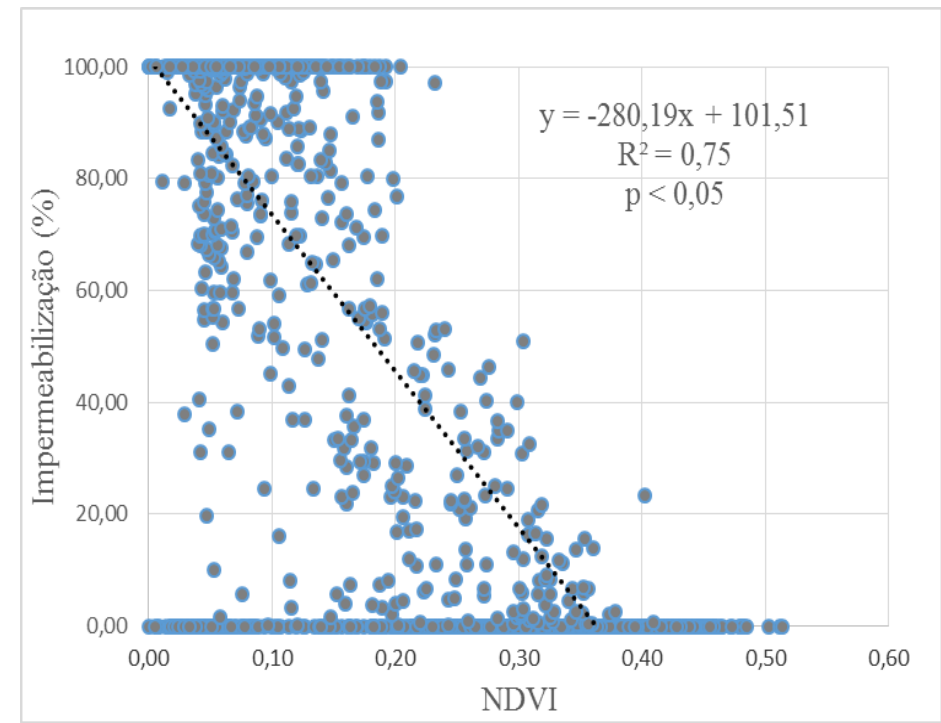

Figura 2 - Reta de regressão construida comparando os valores de NDVI com os valores de impermeabilização do solo extraidos de fotografias aéreas de alta resolução.

Uma caracterisitica interessante que chama a atenção neste gráfico é que, tanto na base quanto no topo do gráfico, observa-se pontos contiguos formando uma linha reta. Estes pontos referem-se às localidades onde foram observadas variações nos valores de NDVI, porém não foram constatadas por meio das fotografias aéreas alterações significativas nos níveis de impermeabilização. As variações de NDVI na base (entre 0 e 0,5 ), que correspondem a $0 \%$ impermeabilização, foram mais intensas que no topo (entre 0 e 0,2 ), correspondendo a $100 \%$ de impermeabilização. Essa variações dos valores de NDVI sem alterações no nivel de impermeabilização se devem em razão da grande diversidade espectral dos materiais presentes na trama urbana. Apesar destes problemas, os pontos distribuidos ao logo da linha diagonal revelaram que o NDVI foi capaz de explicar boa parte das variações relacionadas ao revestimento do solo.

Com relação à imagem que representa a porcentagem de materiais impermeáveis presentes no pixel de 30 metros, observou-se resultados bastante interessantes e condizentes com a estrutura urbana da Região Metropolitana de São Paulo (RMSP). Analisado os dados estatísitcos, é possível afirmar que $80 \%$ da imagem foi modelada de acordo com as expectativas, ou seja, áreas impermeáveis foram tratadas como 
superfícies impermeáveis e áreas permeáveis como superfície permeáveis. Os $20 \%$ restantes foram atribuidos a erros de modelagem. Erro esses cujos valores ficaram fora do intervalo de $0-100 \%$.

A maior parte dos erros foram valores negativos observados em áreas rurais e com relevo mais ondulado. O sombreamento causado pela disposição do relevo foi a principal causa de erro. Não se observou valores negativos expressivos dentro da manacha ubana. Por outro lado, valores acima de $100 \%$ foram observados principalmente nos grandes corpos d'agua em razão provalmente da falta de amostras de controle nestas localizadades, o que resultou na extrapolação das estimativas. Para evitar interpretações equivocadas desses resultados, procedeu-se o reescalonamento dos valores conforme as descrições já apresentadas na metodologia.

No final desse processo, observou-se uma variação contínua nos valores de impermeabilização do solo seguindo uma distribuição bimodal com um ligeiro dominio de pixel (cerca de 65\%) com valores abaixo de 50\% de impermeabilização. Tal fato decorre da área de estudo também envolver áreas rurais, represas, matas etc. Restringindo rapidamente a análise dentro da mancha urbana, com frequencia, o nível de impermeabilização superou a taxa de $80 \%$.

A Figura 3 ilustra o resultado final do mapeamento, onde os níveis de impermeabilização do solo são distinguidos de acordo com a intensidade de níveis de cinza. Em outras palavras, quanto maior a impermeabilização, mais claro é o pixel da imagem. Conforme pode ser observado, a mancha urbana foi bem destacada das áreas rurais e dos corpos d'água. Especialmente na franja urbana, é possivel observar tonalidades intermediárias que denotam mistura de materiais permeáveis e impermeáveis, o que é de se esperar, uma vez que se trata de áreas de expansão ou de transição entre o urbano e rural. Dentro do continuo urbano, como regra geral, observa-se altas porcentagens de impermeabilização (acima de 50\%) que são interrompidas pela presença especialmente de arborizações nas ruas e parques. Portanto, as áreas de menor impermeabilização neste mapeamento também correspondem aos bairros que teoricamente são mais valorizados. Na porção oeste, dentro do continuo, observa-se, por exemplo, uma mancha mais expressiva de tonalidade média onde estão localizados os bairros Jardins, Morumbi e também o campus principal da Universidade de São Paulo.

Um outro ponto que merece ser destacado é a nitiez com que o sistema viário foi realçado. A linha branca que se destaca formando um arco na porção sul da imagem corresponde o trecho sul do Rodoanel Mário Covas (oficialmente SP-021).

Erros também foram observados na estimativa e merecem algumas considerações. $\mathrm{O}$ principal erro encontrado no modelo foi considerar classes permeáveis de alto albedo como superfície impermeável. Estas classes (solo exposto preparado para o cultivo, loteamentos, entornos das represas, pedreiras etc.) 


$\begin{aligned} & \text { XVII Simpósio Brasileiro } \\ & \text { de Geografia Fisica Aplicada }\end{aligned}$
$\begin{aligned} & \text { I Congresso Nacional } \\ & \text { de Geografia Física }\end{aligned}$

foram modeladas em sua maioria como sendo $100 \%$ impermeáveis. Os casos mais evidentes deste erro são encontrados nas margens dos grandes reservatórios de água que circundam a RMSP (Alto Cotia, Guarapiranga, Billings, Paiva Castro e Taiaçupeba). Em razão do rebaixamento expressivo dos níveis de água dessas represas em decorrência da forte seca que atingiu a região no ano de 2015, observou-se em suas margens extensas faixas contínuas de solo exposto antes cobertos pelas águas.

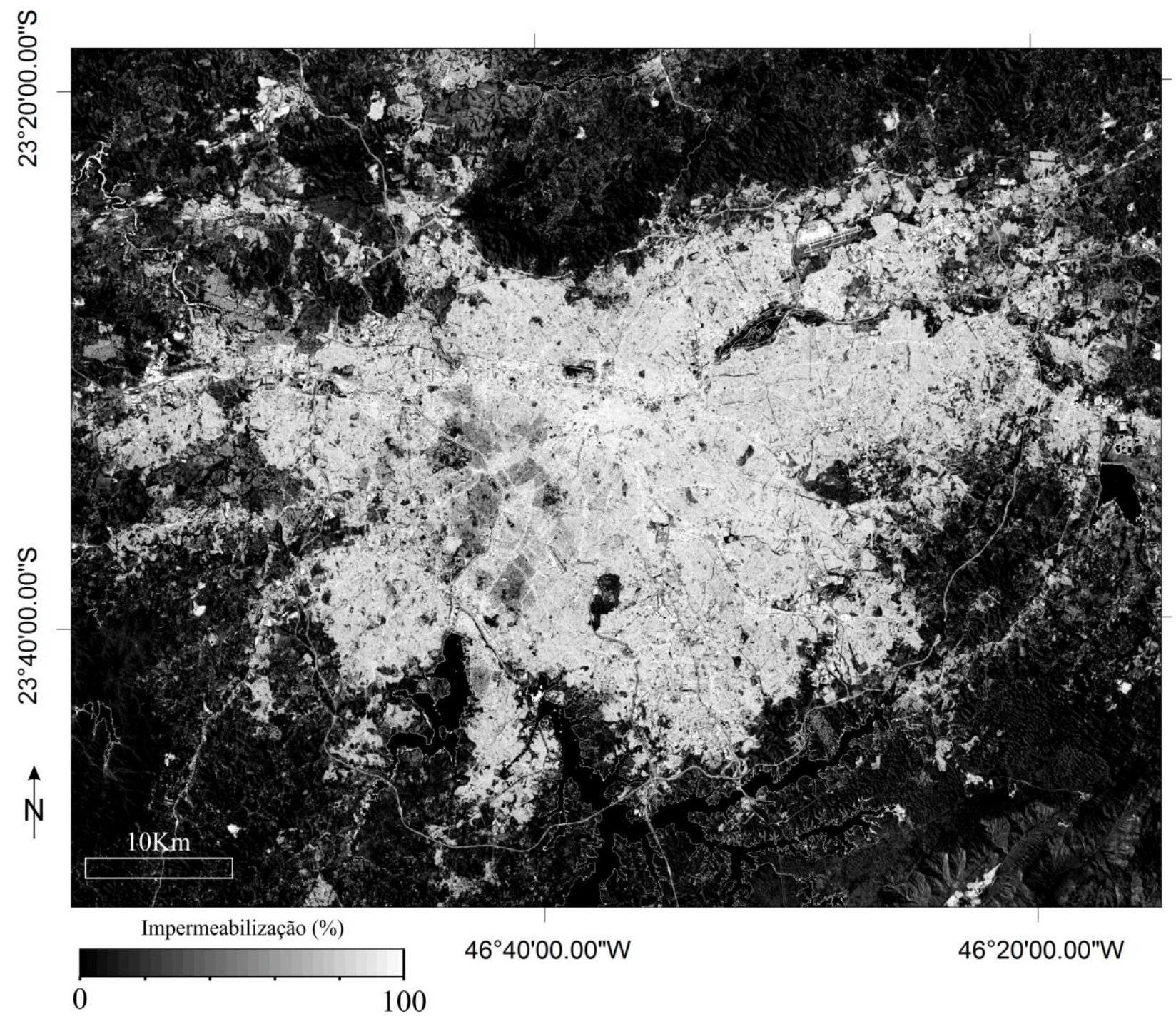

Figura 3 - Estimatimativa de superfície impermeável por meio da regressão linear utilizando como variável explicativa o NDVI derivado das imagens OLI Landsat-8. A imagem possui dimensão de 2500 colunas por 2000 linhas.

Conforme já mencionado, a validação dos resultados também foi feita de maneira quantitativa por meio da técnica de validação cruzada. Ou seja, comparando os valores estimados com amostras de referências tidas como "reais". A igualdade nos valores correspodem aos acertos e a diferença, os erros, que podem ser 


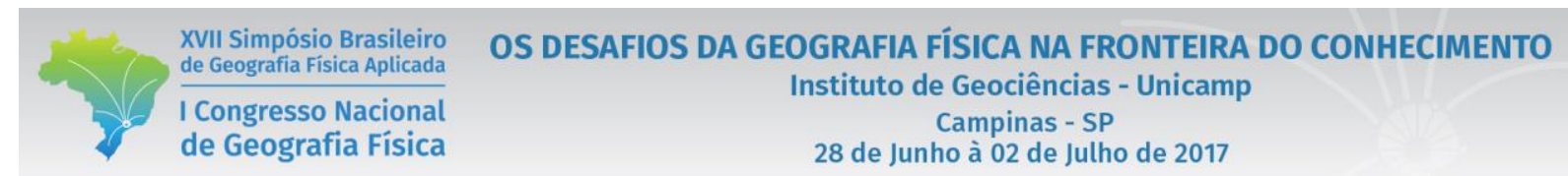

significativos ou não. A Figura 4 ilustra o gráfico de dispersão com o respectivo ajuste linear. Conforme pode ser observado, o valor do $\mathrm{R}^{2}$ foi de $0,89(p<0,05)$, evidenciando, portanto uma boa estimativa do modelo. Observa-se, porém, que a nuvem de pontos não se distribue na forma de uma elipse ao longo da reta, o que levanta a suspeita de que o erro associado pode não seguir um modelo de distribuição normal. Esta suspeita foi confirmada com o teste de normalidade Shapiro-Wilk com valor de $p<0,05$.

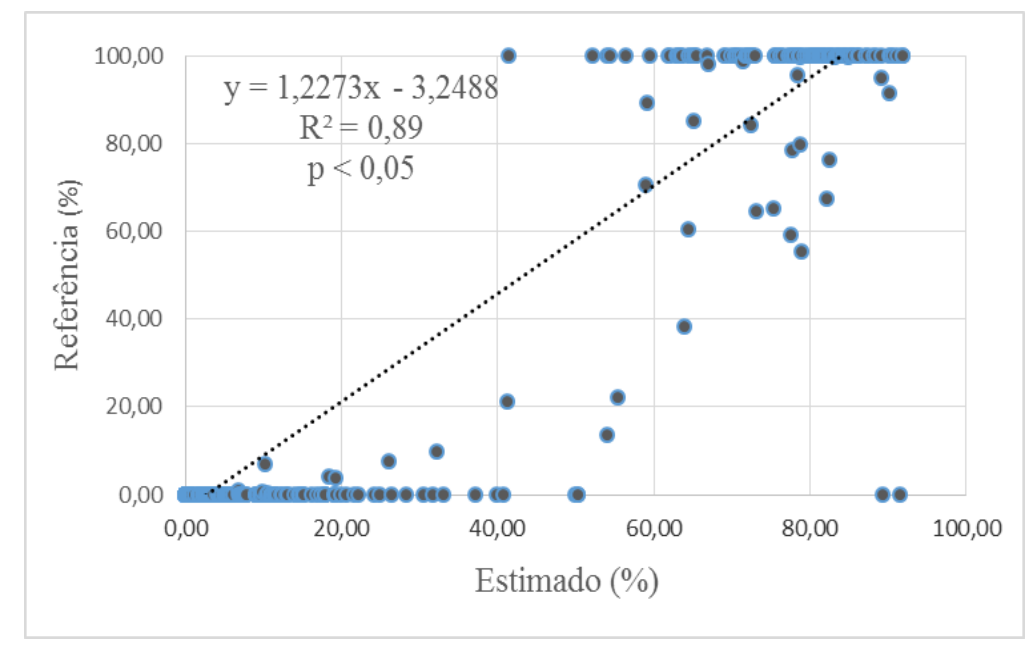

Figura 4 - Estimatimativa de superfície impermeável por meio da regressão linear.

A Figura 5 ilustra o histograma do resíduo. A média encontrada do resíduo foi 2,7 com desvio padrão de 16,2. Portanto, a análise do resíduo também revelou que o valor real foi ligeiramente inferior ao estimado, evidenciando um pequeno erro de superestimativa do grau de impermeabilização do solo.

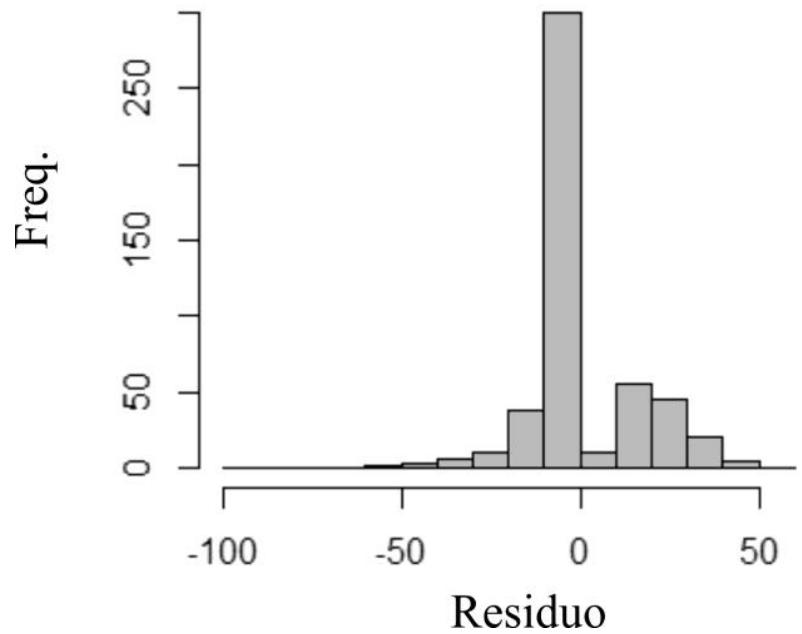

Figura 5 - Estimatimativa de superfície impermeável por meio da regressão linear. 


\section{Conclusões}

Os resultados alcançados no presente estudo mostram a eficiência do método de regressão linear para predizer o grau de impermeabilização do solo tendo como variável explicativa os valores do índice de vegetação NDVI. Observou-se correlação estatísitica $\mathrm{R}^{2}$ igual a $0,89(p<0,05)$ ao comparar o valor real com o estimado. O principal erro encontrado na modelagem foi considerar áreas de solo exposto como superfícies impermeáveis. A análise de resíduo mostrou que a superfície estimada é ligeiramente superior ao real, e como consequência, cuidados devem ser tomados ao utilizar a superfície como parâmetros de entrada para a modelagem de escoamento superficial e de qualidade das águas.

\section{Bibliografia}

ARNOLD, C. L.; GIBBONS, C. J. Impervious Surface Coverage: The Emergence of a Key Environmental Indicator. Journal of the American Planning Association, v. 62, n. April 2012, p. 243-258, 1996.

CANTERS, F.; Chormanski, J.; Voorde , T. V.; Batelaan, O. Effects of different methods for estimating impervious surface cover on runoff estimation at catchment level. In: 7 TH INTERNATIONAL SYMPOSIUM ON SPATIAL ACCURACY ASSESSMENT IN NATURAL RESOURCES AND ENVIRONMENTAL SCIENCES. Anais...Lisboa: Universidade Nova de Lisboa, 2006. p. 557-566.

CERON, A. O.; DINIZ, J. A. F. O Uso das fotografias aéreas na identificação das formas de utilização agrícola da terra. Revista Brasileira de Geografia, v. 2, p. 161-173, 1966.

CHANGNON, S. A. Inadvertent weather modification in urban areas: lessons for global climate change. Bull. Am. Meteorol. Soc., v. 73, p. 619-627, 1992.

PAINE, D.; KISER, J. D. Aerial photography and image interpretation. NJ: John Wiley \& Sons, 2003.

SCHUELER, T. R. The Importance of Imperviousness. Watershed Protection Techniques, v. 1, n. 3, p. 100-111, 1994.

TOCCI, C. E. M. Água no meio urbano. Porto Alegre: UFRGS, 1997.

WENG, Q. Remote Sensing of Impervious Surfaces. Boca Raton: CRC Press, 2007. 


$\begin{aligned} & \text { XVII Simpósio Brasileiro } \\ & \text { de Geografia Fisica Aplicada }\end{aligned}$
$\begin{aligned} & \text { I Congresso Nacional } \\ & \text { de Geografia Física }\end{aligned}$

YUAN, F.; BAUER, M. E. Comparison of impervious surface area and normalized difference vegetation index as indicators of surface urban heat island effects in Landsat imagery. Remote Sensing of Environment, v. 106, n. 3, p. 375-386, 2007. 